\title{
Effects of receptor modification and temperature on dynamics of sensory complexes in Escherichia coli chemotaxis
}

\author{
Sonja Schulmeister ${ }^{1,2}$, Karin Grosse ${ }^{1}$ and Victor Sourjik ${ }^{1 *}$
}

\begin{abstract}
Background: Extracellular stimuli in chemotaxis of Escherichia coli and other bacteria are processed by large clusters of sensory complexes. The stable core of these clusters is formed by transmembrane receptors, a kinase CheA, and an adaptor CheW, whereas adaptation enzymes CheR and CheB dynamically associate with the clusters via interactions with receptors and/or CheA. Several biochemical studies have indicated the dependence of the sensory complex stability on the adaptive modification state of receptors and/or on temperature, which may potentially allow environment-dependent tuning of its signalling properties. However, the extent of such regulation in vivo and its significance for chemotaxis remained unclear.

Results: Here we used fluorescence recovery after photobleaching (FRAP) to confirm in vivo that the exchange of CheA and CheW shows a modest dependency on the level of receptor modification/activity. An even more dramatic effect was observed for the exchange kinetics of CheR and CheB, indicating that their association with clusters may depend on the ability to bind substrate sites on receptors and on the regulatory phosphorylation of CheB. In contrast, environmental temperature did not have a discernible effect on stability of the cluster core. Strain-specific loss of E. coli chemotaxis at high temperature could instead be explained by a heat-induced reduction in the chemotaxis protein levels. Nevertheless, high basal levels of chemotaxis and flagellar proteins in common wild type strains MG1655 and W3110 enabled these strains to maintain their chemotactic ability up to $42^{\circ} \mathrm{C}$.

Conclusions: Our results confirmed that clusters formed by less modified receptors are more dynamic, which can explain the previously observed adjustment of the chemotaxis response sensitivity according to the level of background stimulation. We further propose that the dependency of CheR exchange on the availability of unmethylated sites on receptors is important to improve the overall chemotaxis efficiency by suppressing molecular noise under conditions of high ligand concentrations. Moreover, the observed stability of the cluster core at high temperature is in line with the overall thermal robustness of the chemotaxis pathway and allows maintenance of chemotaxis up to $42^{\circ} \mathrm{C}$ in the common wild type strains of E. coli.
\end{abstract}

\section{Background}

Chemotaxis enables motile bacterial cells to follow environmental chemical gradients, migrating towards higher concentrations of attractants while avoiding repellents. Despite some deviations in protein composition, all studied bacterial chemotaxis systems rely on a similar strategy of following chemical gradients, using

\footnotetext{
* Correspondence: v.sourjik@zmbh.uni-heidelberg.de

'Zentrum für Molekulare Biologie der Universität Heidelberg, DKFZ-ZMBH

Alliance, Im Neuenheimer Feld 282, 69120 Heidelberg, Germany

Full list of author information is available at the end of the article
}

the same conserved core of signaling proteins. The pathway in Escherichia coli is the best-studied model, see $[1,2]$ for recent reviews. Sensing and processing of stimuli in bacterial chemotaxis is performed by complexes that consist of several attractant-specific chemoreceptors, a histidine kinase CheA, and an adaptor protein $\mathrm{CheW}$. Attractant binding to the periplasmic part of a receptor rapidly inhibits CheA autophosphorylation, reducing phosphotransfer to the motor regulator $\mathrm{CheY}$ and thereby promoting smooth swimming. This initial rapid response is followed by slower adaptation, which

\section{Biomed Central}


is mediated by methylation of receptors on four specific glutamate residues by a methyltransferase CheR. The inverse reaction of receptor demethylation is mediated by the methylesterase CheB. Receptors are originally expressed in a half-modified state (QEQE), where glutamines $(\mathrm{Q})$ mimic the effects of methylated glutamates and are deamidated by CheB. Higher modification of receptors increases activity of the associated CheA and lowers receptor sensitivity to attractants, thereby allowing cells to adapt to a persistent attractant stimulus [3-9]. The feedback from the sensory complex activity to the methylation system is believed to come primarily from the substrate specificity of adaptation enzymes, with CheR preferentially methylating inactive receptors and $\mathrm{CheB}$ preferentially demethylating active receptors [10-12]. An additional negative feedback is provided by the CheA-mediated phosphorylation of $\mathrm{CheB}$, which increases CheB activity but is not essential for chemotaxis [13] and has little effect on the kinetics of adaptation to positive stimuli $[10,14,15]$.

Although in vitro experiments suggest that the transmembrane signal transduction and kinase regulation in chemotaxis could be performed by small receptor-kinase complexes that consist of two to three receptor dimers, several CheW and one CheA molecules [11,16-18], in the cell the receptor-kinase sensory complexes are organized into macromolecular clusters that can contain thousands of receptors and associated chemotaxis proteins. Larger clusters typically localize at the cell poles, while several smaller clusters are found along the cell body [19-21]. In these clusters, receptors are arranged in roughly hexagonal arrays that are presumably formed by trimers of receptor homodimers [22-25], with different receptors able to form mixed trimers [26]. Clusters are further stabilized by the association of CheA and/or CheW [19,20,27-29]. Receptor clusters are important for signal processing in chemotaxis, whereby allosteric interactions between receptors within clusters allow amplification and integration of chemotactic signals [7,30-33]. All other chemotaxis proteins - CheR, CheB, CheY and CheZ - localize to receptor clusters in E. coli through association with either receptors (CheR) or CheA (CheZ and CheY) or both (CheB) [20,34-36]. Receptor clustering plays therefore an additional role by providing a scaffold for chemotaxis signalling [2]. The relatively stable signal-processing core of these clusters is composed of receptors, CheA, CheW and a phosphatase CheZ, along with the dynamically exchanging adaptation enzymes and CheY [37]. Adaptation enzymes are believed to primarily localize to the clusters via association with the $\mathrm{C}$-terminal pentapeptide sequence of major receptors Tar and Tsr [35,36,38-40], but they also bind to their substrate sites - unmethylated glutamates for CheR and glutamines or methylated glutamates for
$\mathrm{CheB}$ - on the receptors. Moreover, $\mathrm{CheB}$ also binds to the P2 domain of CheA, competing for the binding site with CheY $[40,41]$.

The aim of this study was to investigate whether cluster stability in vivo is regulated by such physiologically relevant factors as adaptation to the chemotactic signals and by the environmental temperature. Several biochemical studies indicated that stability of sensory complexes might strongly increase with the level of receptor methylation [7,42]. However, a more recent study reported extreme ultrastability of the biochemically reconstituted sensory complexes with no discernible effect of receptor modification under the reference conditions [43], although complexes formed by the less modified receptors did show higher susceptibility to destabilizing agents. Surprisingly, this later study also reported a dramatic reduction of the complex stability at temperatures above $30^{\circ} \mathrm{C}$. By performing an in vivo analysis of cluster stability using fluorescence recovery after photobleaching (FRAP), we were able to reconcile these apparently conflicting biochemical studies by showing that the exchange of CheA and CheW at receptor clusters is weakly dependent on the receptor modification. Our results also suggest a strong dependence of the exchange rates of adaptation enzymes on their ability to bind substrate sites on receptors, and on CheB phosphorylation. We propose that both effects play an important role in the overall strategy of bacterial chemotaxis. Moreover, in line with the recently described thermal robustness of the chemotaxis pathway [44] we observed that stability of the cluster signalling core is not affected by temperature and that the common wild type $E$. coli strains can perform chemotaxis up to $42^{\circ} \mathrm{C}$.

\section{Results}

\section{Receptor modification affects stability of the cluster core}

To test effects of receptor modification on the exchange dynamics of CheW and CheA at receptor clusters, FRAP experiments were performed in an adaptationdeficient $(\Delta$ cheRcheB $)$ strain and in the $\mathrm{CheR}^{+} \mathrm{CheB}^{+}$ strain. In the former strain, receptors are present in their original half-modified (QEQE) state, which leads to a nearly maximal activation of the associated CheA in vivo $[5,8,32]$. In contrast, in the adapted $\mathrm{CheR}^{+} \mathrm{CheB}^{+}$ strain the average level of receptor modification and activity are significantly lower $[5,8,32,44]$ (see also additional file 1, Figure S1). To facilitate FRAP experiments, both strains carried an additional deletion of the negative regulator of late flagellar and chemotaxis gene expression, anti-sigma factor FlgM. This deletion leads to an approximately 6-fold overexpression of all chemotaxis genes and consequently to larger clusters, without any negative effects on chemotactic performance $[37,45]$. 
FRAP experiments were performed as previously described [37], whereby the fluorescence was bleached by two short laser pulses in the polar region of the cell, and subsequent recovery of relative fluorescence at the pole was followed over time (see Methods for details). As in this previous study, we used the C-terminal fusion of yellow fluorescent protein to $\mathrm{CheW}$ (CheW-YFP) and the $\mathrm{N}$-terminal fusion to a truncated form of CheA that lacks first 258 amino acids (YFP-CheA ${ }^{\Delta 258}$ ). The latter fusion was chosen because it has a more clear localization pattern to receptor clusters than YFP fusion to the full-length CheA $\left(\mathrm{CheA}_{\mathrm{L}}\right)$ or the natively occurring short version of CheA $\left(\mathrm{CheA}_{\mathrm{S}}\right)$. Notably, all CheA fusions and both $\mathrm{N}$ - and $\mathrm{C}$-terminal CheW fusions showed similar exchange kinetics in previous FRAP experiments, suggesting that the exchange kinetics at the cluster is unaffected by the YFP fusion [37]. Consistent with that, CheW-YFP fusion has been shown to form ultrastable ternary complexes in vitro, similar to those formed by the untagged CheW [43].

Thus obtained recovery kinetics was clearly biphasic for all fusions (Figure 1). Our previous detailed analysis of FRAP data demonstrated that the initial phase of fast recovery corresponds to the exchange of the freely diffusing fusion protein in the region of interest, whereas the second phase specifically reflects protein exchange at the cluster [37]. In this study, we therefore were only interested in this slow phase of recovery, which reflects the concentration-independent rate constant of protein dissociation from the cluster $\left(k_{o f f}\right)$ [37].
Analysis of obtained recovery kinetics showed that the exchange of CheA, and to a lesser extent of CheW, was slower in $\Delta$ cheRcheB strain than in the $C^{2} \mathrm{R}^{+}$ $\mathrm{CheB}^{+}$strain (Figure 1a, b). Whereas in the Che $\mathrm{R}^{+}$ $\mathrm{CheB}^{+}$strain the characteristic turnover time $\left(k_{o f f}{ }^{-1}\right)$ of CheA at the cluster was $15 \mathrm{~min}$, as observed before [37], little recovery was observed in the $\Delta$ cheRcheB strain even after $20 \mathrm{~min}$. This strongly suggests that receptors with higher levels of modification (and therefore higher activity) form signalling complexes that are more stable.

To further test whether the level of modification directly affects the exchange of receptors at the cluster, we performed FRAP experiments on YFP fusions with two extreme modification states of an aspartate receptor Tar - fully unmodified Tar ${ }^{\text {EEEE }}$ and fully modified Tar ${ }^{\mathrm{QQQ}}$. These fusions were tested in $\triangle$ cheRcheB background, which also expresses the original untagged receptors in the half-modified state. This was necessary because YFP-tagged receptors do not form clusters very efficiently when expressed alone, presumably due to perturbing effects of multiple fluorescent proteins on the cluster structure. Little exchange was observed in this experiment even for the fully unmodified receptors (Figure 1c), suggesting that even inactive receptors are stably incorporated into the receptor clusters. The faster exchange of CheA at the clusters of less modified receptors is therefore likely to reflect the dynamics of kinase association with receptors rather than the exchange of receptors themselves.
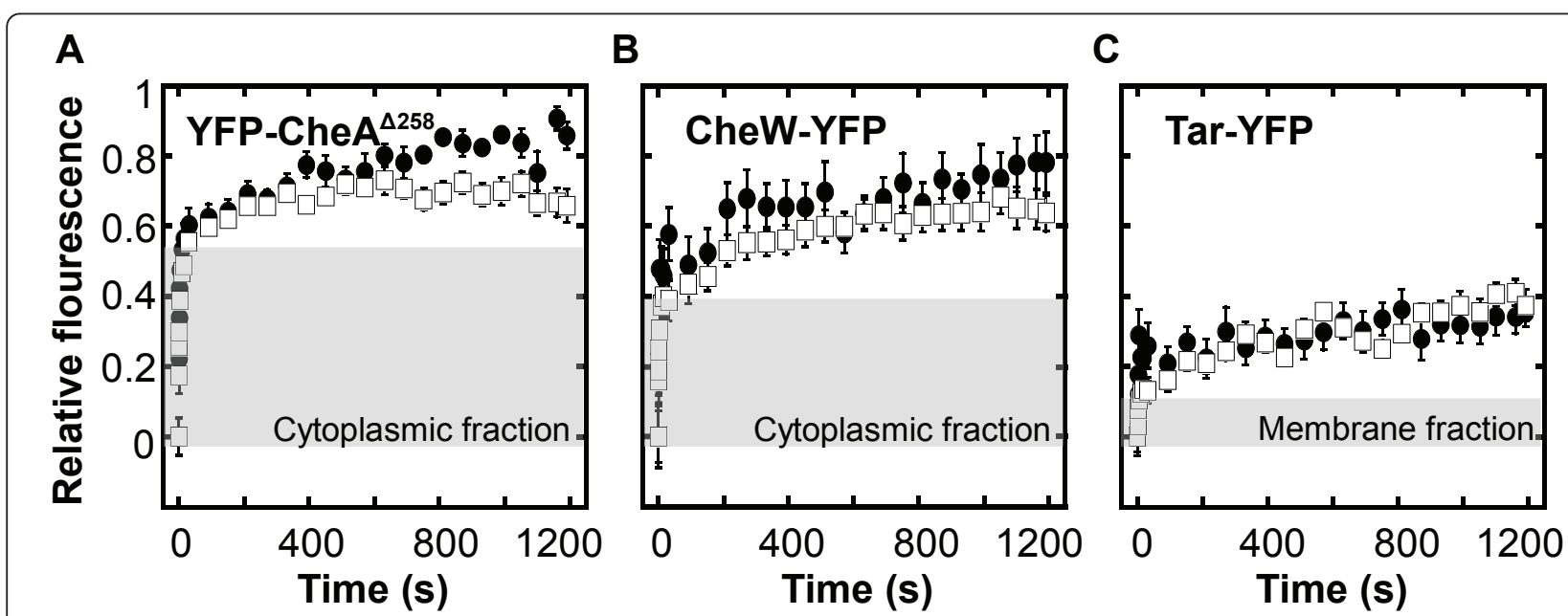

Figure 1 Protein exchange at the cluster core. (a-b) Recovery of YFP-CheA $A^{\Delta 258}$ (a) and CheW-YFP (b) in strain LL4 (CheR $\left.R^{+} C h e B^{+}\right)$where receptors are in the low modification state (filled circles) and in strain LL5 ( $\triangle$ cheR $\triangle c h e B$ ) where receptors are in the intermediate modification state (white squares). (c) Recovery of unmodified Tar ${ }^{E_{E E E}}$-YFP (filled circles) and fully modified Tar ${ }^{\mathrm{QQQQ}}$-YFP (white squares) receptors in strain LL5. Curves represent means of 14 to 27 experiments, with error bars indicating standard errors. To reduce variability associated with the varying depth of bleaching, the value of the first post-bleach point was subtracted prior to normalization to the relative intensity before photobleaching (see Methods). Grey shading indicates the initial rapid recovery of the fusion protein that is not incorporated into the cluster and freely diffuses in the cytoplasm or in the plasma membrane (see text). 


\section{Receptor modification and pathway activity affect exchange of adaptation enzymes}

We next investigated whether the dynamics of the adaptation enzymes at the cluster might be regulated at the level of the receptor modification and/or the pathway activity. As mentioned above, Che R and CheB bind not only to the C-terminal pentapeptide sequence of receptors but also to their substrate sites on receptors unmethylated glutamates and glutamines or methylated glutamates, respectively. Consistent with the significant contribution of the binding of CheR and CheB to their substrate sites to the overall exchange dynamics, we observed a clear increase in the exchange rates of CheR (Figure 2a) and CheB (Figure $2 \mathrm{~b}$ ) in strains where this binding was compromised. Whereas the characteristic exchange time of CheR in $\mathrm{CheR}^{+} \mathrm{CheB}^{+}$cells was $\sim 15$ $\mathrm{sec}$, this time was reduced to $\sim 6 \mathrm{sec}$ in the strain that lacks $c h e B$, thus having all receptors in a fully modified state (i.e., QEmQEm, where Em is the methylated glutamate), with no substrate sites available for methylation (Figure 2a and Figure S1a). A very similar reduction has been observed for the catalytic mutant of CheR $\left(C_{e} R^{\mathrm{D} 154 \mathrm{~A}},[36]\right)$ in $\Delta$ cheRcheB cells (Figure 2a). Although in these cells receptors are in the half-modified (QEQE; Figure S1a) state and thus have available substrate sites, the catalytic mutant of CheR apparently fails to bind to these sites efficiently. The dependence of Che R exchange on the level of receptor modification is thus likely to be a direct consequence of its binding to the substrate sites, although it is still possible that

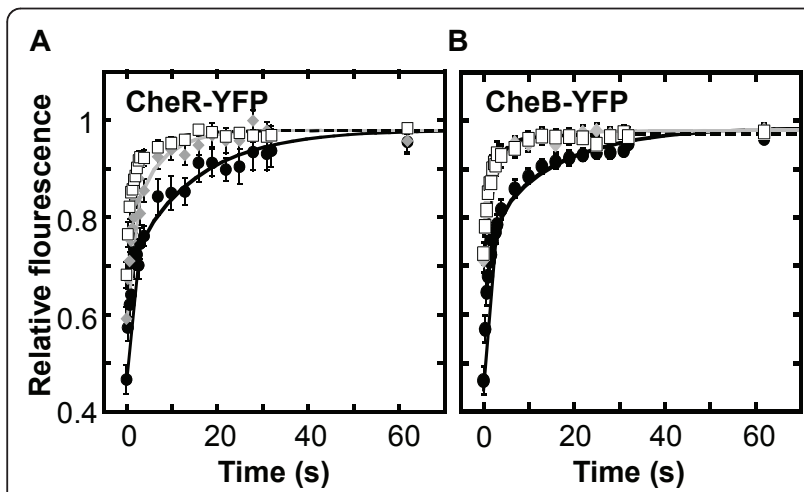

Figure 2 Exchange kinetics of adaptation enzymes. (a) Recovery kinetics of CheR-YFP in strain VS102 (CheR ${ }^{+} \mathrm{CheB}^{+}$) with receptors in low methylated state (filled circles, solid black line; data taken from [37]) and in strain LL5 that lacks chromosomal CheR and CheB (white squares, dashed black line), and recovery kinetics of YFPCheR ${ }^{\mathrm{D} 154 \mathrm{~A}}$ (gray diamonds, gray line) in strain LL5. (b) Recovery kinetics of CheB-YFP in strain VS102 (filled circles, solid black line, data taken from [37]), and of CheB ${ }^{\text {S164C }}$-YFP (gray diamonds, gray line) and CheB ${ }^{\mathrm{D} 56 \mathrm{E}_{-}}$YFP (white squares, dashed black line) in LL5. Curves represent means of 13 to 30 experiments, with error bars indicating standard errors. receptor modification has an indirect, allosteric effect on the affinity of CheR binding.

Similarly, the characteristic exchange time for $\mathrm{CheB}$ was reduced from $\sim 16 \mathrm{sec}$ to $\sim 4 \mathrm{sec}$ upon mutation of the catalytic site $\left(\mathrm{CheB}^{\mathrm{S} 164 \mathrm{C}}\right.$, [46]; Figure $\left.2 \mathrm{~b}\right)$, suggesting that the binding to the substrate sites is similarly important for the overall stability of $\mathrm{CheB}$ association with the cluster. A similar reduction in the exchange time, to $\sim 2.5 \mathrm{sec}$, was observed upon mutating the phosphorylation site of $\mathrm{CheB}\left(\mathrm{CheB}^{\mathrm{D} 56 \mathrm{E}}\right.$; Figure $\left.2 \mathrm{~b}\right)$, consistent with a previous observation that unphosphorylated $\mathrm{CheB}$ shows weaker binding to receptor clusters [40]. Surprisingly, the exchange rate of the wild type CheB in the cheR background was similar to that in the $\mathrm{CheR}^{+} \mathrm{CheB}$ ${ }^{+}$strain (data not shown). We observed, however, that receptors were not fully deamidated in this strain (Figure S1b), likely providing sufficient number of substrate binding sites (Qs) for CheB molecules.

\section{In vivo stability of the cluster core is not affected by temperature}

Finally, we have analyzed effects of temperature on stability of the cluster core. E. coli K-12 strain RP437, used as a wild type in most studies of chemotaxis, as well as its parent strain B275, are known to lose their motility and chemotaxis above $37^{\circ} \mathrm{C}$ (Figure 3a-c) [47]. It was recently proposed that temperature sensitivity of chemotaxis may be related to the observed low stability of biochemically reconstituted chemosensory complexes at high temperature [43]. However, we observed that common wild type E. coli K-12 strains MG1655 and W3110 remain chemotactic up to $42^{\circ} \mathrm{C}$ (Figure 3a-c), despite having the same chemotaxis machinery as RP437. Consistent with that, the intracellular stability of receptor clusters, accessed by the dynamics of CheA exchange, showed no apparent decrease in stability at high temperature (Figure 3d).

These results suggest the downregulation of the chemotaxis gene expression as the most likely cause of the chemotaxis loss in RP437 at high temperature, consistent with the originally favoured explanation [47]. Indeed, under our growth conditions the expression of both major chemoreceptors, Tar and Tsr, was at least 10 times lower at $42^{\circ} \mathrm{C}$ than at $34^{\circ} \mathrm{C}$ (Figure $3 \mathrm{e}$ ), which is likely to reflect a general temperature effect on expression of all chemotaxis and flagellar genes in E. coli. Notably, a similar reduction in the receptor levels was observed in all strains, demonstrating that the effect is not specific to the RP437-related strains. However, since the levels of chemotaxis proteins are generally much higher in MG1655 and W3110, these strains can apparently maintain sufficient expression even at $42^{\circ} \mathrm{C}$, whereas protein levels in RP437 readily drop below the level that is necessary 

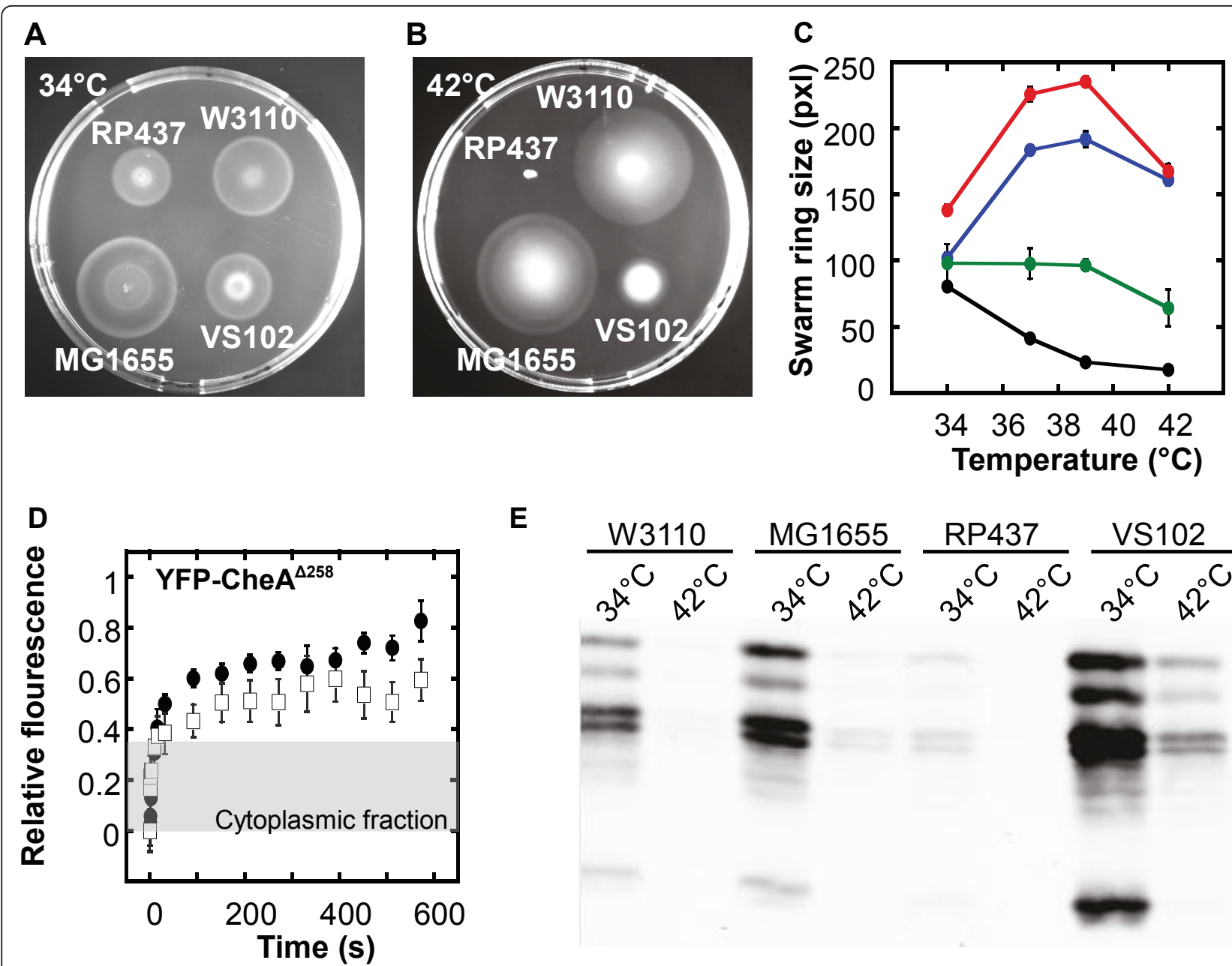

E

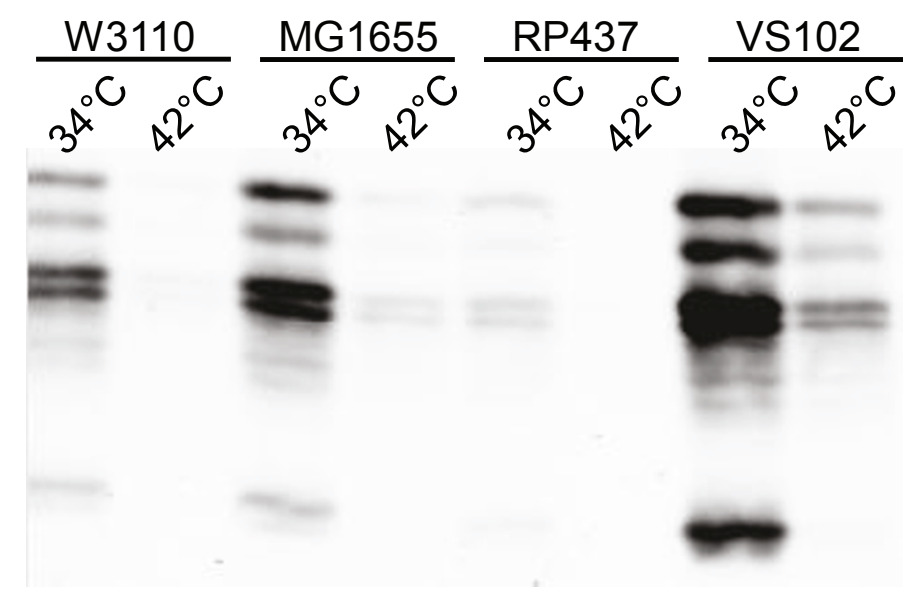

Figure 3 Effects of temperature on chemotaxis and cluster stability. (a-b) Effects of incubation temperature on swarming ability of E. coli strains. Representative swarm plates show swarm rings formed by indicated strains at $34^{\circ} \mathrm{C}$ (a) and $42^{\circ} \mathrm{C}$ (b) after 5 hours. (c) Corresponding swarming efficiency at a function of temperature for strains RP437 (filled circles), W3110 (white squares) and MG1655 (white circles). Standard errors are indicated. (d) Exchange of YFP-CheA ${ }^{\Delta 258}$ at receptor clusters in strain VS102 at $20^{\circ} \mathrm{C}$ (filled circles, data from [37]) and at $39^{\circ} \mathrm{C}$ (white squares). Means of 10 to 20 experiments are shown. Error bars represent standard errors. Grey shading is as in Figure 1. (e) Temperature effects of expression levels of chemotaxis proteins, represented here by chemoreceptors. Expression was detected by immunoblotting as described in Methods using $\alpha$ Tar antibody that also recognizes well other chemoreceptors. In $\mathrm{CheR}^{+} \mathrm{CheB}^{+}$strains used here, each receptor runs as several bands corresponding to different states of modification. See Figure S1 for assignment of individual bands.

for chemotaxis [37,45]. This explanation is further supported by the observation that a substantial degree of chemotaxis was retained at $42^{\circ} \mathrm{C}$ in the RP437-derived $\triangle$ flgM strain VS102, which has elevated levels of all chemotaxis proteins (Figure 3e). Nevertheless, the improvement of chemotaxis at high temperatures was less pronounced in VS102 than in MG1655 and W3110, despite comparatively higher chemotaxis protein levels in the former strain. This suggests that the effect of high temperature cannot be solely compensated by the overexpression of chemotaxis proteins, probably because the low expression of early flagellar proteins, which are not upregulated in VS102, becomes limiting in this case.

\section{Discussion}

Stimulation-dependent regulation of assembly and stability of sensory complexes can be important in signalling, and many signal transduction pathways in eukaryotes are regulated on this level [48]. Here we show that protein exchange at the sensory complexes in $E$. coli chemotaxis is affected by the signalling state of the pathway on many levels. First, stability of the sensory receptorkinase core is higher for complexes formed by receptors that are in a higher modification state and consequently are more active. Such dependence is generally consistent with previous biochemical experiments $[7,42]$, with lower structural stability of less modified receptors [49], and also with higher sensitivity of sensory complexes 
that are formed in vitro by the less modified receptors to destabilizing factors such as high $\mathrm{pH}$ or low ionic strength [43]. Our data also agree with in vivo studies that reported an increase in protein localization to the chemoreceptor clusters $[50,51]$ at higher levels of receptor modification or activity. However, the effect in vivo is rather modest, and the observed regulation of complex stability dependent on receptor modification is unlikely to be directly involved in signal transduction. Rather, it may play a role in the adjustment of the signalling properties of receptor clusters, and can indeed explain the previously observed increase in the strength of cooperative receptor interactions within clusters upon increase in receptor modification [5]. Since increased methylation results from adaptation to increasing concentration of ambient attractant, higher stability and cooperativity within clusters can enhance the gain of the chemotaxis system at higher levels of ambient ligands, to closely follow physical limits of sensitivity posed by the noise in ligand binding [5].

The regulation of exchange at the cluster that was observed for the adaptation enzymes may be of even greater physiological significance. When CheR is unable to bind its substrate sites on the receptor, whether due to the mutation in the catalytic site of CheR or lack of unmethylated glutamates, the turnover was greatly accelerated. This suggests that the overall rate of CheR dissociation from receptors $\left(k_{o f f}\right)$ largely depends on its binding to the substrate sites, although such dependence remains to be confirmed by direct biochemical measurements. In principle, the level of receptor modification might also affect this turnover indirectly, through an allosteric regulation of Che R association with the Cterminal pentapeptide sequence of receptors. Regardless of the detailed molecular mechanism of such methylation-dependent acceleration of CheR exchange, we propose that faster turnover can increase the efficiency of adaptation by limiting the amount of time CheR spends in an unproductive association with a receptor molecule that cannot be further modified. This is particularly important for adaptation to high levels of ambient stimulus, when the kinetics and precision of adaptation become severely limited by the shortage of the free methylation sites [15,52].

Another important effect of the faster turnover of Che $\mathrm{R}$ at the cluster may be to specifically reduce the noise in the signalling output at increased levels of receptor methylation. Previous studies suggested that the level of phosphorylated CheY in adapted E. coli cells can vary substantially on the time scale of tens of seconds [53]. This can be explained by stochastic fluctuations in the number of cluster-associated CheR molecules [53-55] that would translate into the variable level of receptor methylation and ultimately into fluctuations of the activity of the pathway. Such fluctuations are expected to result in E. coli cells occasionally undertaking very long runs, enhancing the overall efficiency of the population spread through the environment in the search of chemoattractant gradients $[54,55]$. However, fluctuating levels of CheY-P are also predicted to severely impair the ability of bacteria to precisely accumulate at the source of the chemoattractant gradient, posing a trade-off dilemma for the chemotaxis strategy [55]. We propose that the observed increase in the turnover of CheR at the highly methylated receptors will specifically decrease noise in the pathway output for cells that have already reached high attractant concentration along the gradient, enabling them to efficiently accumulate at the source of attractant. The observed regulation of CheR exchange may therefore be an evolutionary selected trait that increases overall chemotaxis efficiency.

An acceleration of exchange was also observed for the catalytic mutant of CheB. This indicates that the CheB exchange is dependent on its binding to substrate sites, similar to CheR, though the molecular details of this effect remain to be clarified. Moreover, CheB exchange was strongly stimulated by mutating the phosphorylation site in the regulatory domain, which prevents $C h e B$ activation by phosphorylation. This latter effect confirms that the binding of $\mathrm{CheB}$ to receptor clusters is strengthened by phosphorylation, which may provide an additional regulatory feedback to the chemotaxis system ([40]; Markus Kollmann, personal communication).

Finally, we analyzed here the effects of temperature and showed that the thermal stability of the cluster core in the cell, determined by the exchange of CheA, is much higher than that of the biochemically reconstituted complexes [43]. While the factors responsible for the observed differences between stability of the sensory complexes in vivo and in vitro remain to be identified, such differences may be due to the active process of assembly and/or disassembly of the sensory complexes by specialized cellular chaperones as previously proposed [43]. In any case, thermal stability of the cluster core may be an important component of the overall thermal robustness of the chemotaxis pathway [44]. Consistent with that, the deterioration of chemotaxis in some $E$. coli strains above $37^{\circ} \mathrm{C}$ is apparently caused by the reduced expression of chemotaxis and flagellar genes rather than by the malfunction of the pathway. Moreover, although the observed effect of temperature on gene expression was not strain-specific, chemotaxis of the wild type strains MG1655 and W3110 was significantly less affected than chemotaxis of RP437. This difference was apparently due to the generally higher expression of chemotaxis proteins in MG1655 or W3110, which enables these strains to maintain 
expression that is sufficient for chemotaxis up to $42^{\circ} \mathrm{C}$. Thus, the ability to maintain chemotaxis at high temperature is likely to be accomplished by a combination of the thermally robust pathway design [44] with the high thermal stability of chemosensory complexes and high basal expression levels of chemotaxis and flagellar proteins.

\section{Conclusions}

In summary, we observed that the rate of protein exchange at the chemosensory clusters in E. coli depends on the level of adaptive receptor modification. We believe that this dependency may reflect a specific regulatory mechanism to adjust the signalling properties of the chemotaxis system according to varying levels of ambient attractant stimulation, corresponding to two distinct regimes of bacterial chemotaxis that can be described as "searching" and "tracking" behaviour
(Figure 4). Searching behaviour is exhibited by chemotactic bacteria when they explore the environment in the search of attractant gradients in the absence (or at low levels) of ambient ligand. In this regime the level of receptor modification is low, which would result in higher dynamics of the cluster core and slow exchange of CheR at the receptor clusters. The former apparently limits the cooperative interactions between receptors and consequently signal amplification by the clusters. This is physiologically meaningful because sensitivity towards small changes in attractant concentration under these conditions is physically limited by the stochastic noise in ligand binding. The long dwell time of CheR at receptors is also favourable for the explorative behaviour in this regime, because it produces large stochastic fluctuations in the pathway activity over time, thereby promoting faster spread through the environment. The second regime, tracking behaviour, is expected to occur

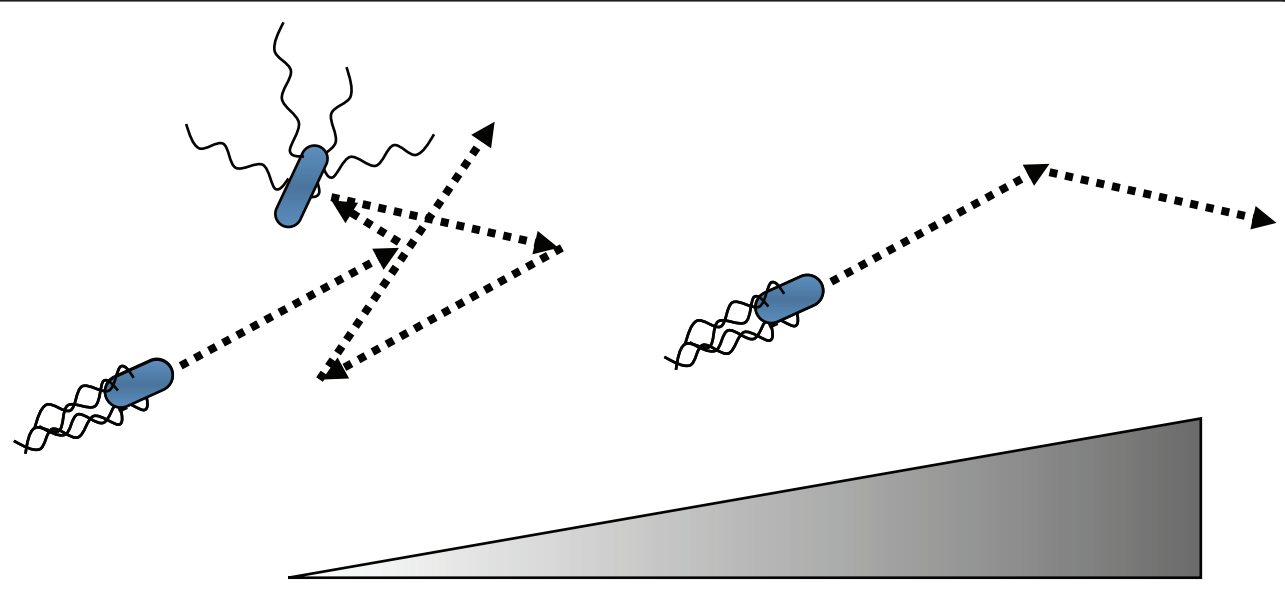

Behaviour

Noise in ligand binding

Receptor methylation

Signal amplification

Output
Tracking

Low

High

Figure 4 Two regimes of bacterial chemotaxis behaviour and their characteristic features. Attractant gradient is indicated. At low concentrations or absence of attractant the behaviour is explorative, while at high concentrations of attractant the behaviour is tracking. See text for details. 
when the cells are moving along the gradient and are already adapted to high ambient concentration of attractant. In that case, the levels of receptor methylation are high, which would increase the dynamics of CheR at the clusters, thereby effectively suppressing the stochasticity of the pathway output and allowing cells to follow the gradient more precisely. High receptor methylation would also enhance cluster stability, leading to stronger amplification of signals under conditions of the reduced ligand binding noise at high concentrations of ambient attractant.

Finally, we demonstrated the thermal stability of the chemosensory complexes in vivo, which may be an important component of the overall thermal robustness of the chemotaxis pathway [44]. This is consistent with the ability of the common wild type E. coli strains to chemotax efficiently up to $42^{\circ} \mathrm{C}$. In these strains, the reduction of the chemotaxis and flagellar gene expression at high temperature is further balanced by high basal levels of the respective proteins, thus ensuring that chemotaxis is supported throughout the entire physiological range of temperatures.

\section{Methods}

\section{Bacterial strains and plasmids}

All strains used for FRAP measurements are derivatives of the E. coli K-12 strain RP437 that is conventionally used as the wild type for chemotaxis studies [56]: VS102 carries a deletion of the anti-sigma-factor flgM, resulting in an approximately 6-fold over-expression of all chemotaxis proteins [45], which has been previously shown to facilitate FRAP measurements of chemotaxis clusters in $E$. coli [37]. LL4 ( $\Delta$ flgM $\Delta$ cheY-cheZ) and LL5 ( $\Delta$ flgM $\Delta$ cheR$c h e Z)$ served as backgrounds corresponding to receptors in low- and intermediate modification state, respectively. In addition, common E. coli $\mathrm{K}-12$ wild type strains MG1655 and W3110 were used as controls for studies of temperature effects on chemotaxis. YFP tagged chemotaxis proteins were expressed from the vector plasmid pTrc99a $\left(A m p^{r}\right)$ under control of an isopropyl- $\beta$-D-thiogalactopyranoside (IPTG) inducible pTrc promoter [57]. Site-specific mutagenesis was used to introduce mutations into catalytic sites of CheR and CheB $[36,40,46]$. As reported previously, YFP fusions to $C$ heR and $C h e B$ are fully functional, whereas fusions to CheA and CheW do not efficiently support chemotaxis but show proper localization to receptor clusters and interactions with their respective binding partners $[36,40,46]$. All expression constructs for the YFP fusions and respective induction levels employed for FRAP are presented in Table 1.

\section{Cell growth and preparation}

For FRAP measurements cells were grown as described elsewhere [37]. In brief, overnight cultures were diluted
Table 1 Plasmids used in this study

\begin{tabular}{|c|c|c|c|}
\hline Plasmid & Relevant genotype & $\begin{array}{c}\text { Induction level } \\
(\mu \mathrm{M} \text { IPTG })\end{array}$ & Reference \\
\hline pDK54 & cheW-eyfp & 50 & {$[37]$} \\
\hline pSS8 & eyfp-cheA ${ }^{-258}$ & 50 & {$[37]$} \\
\hline pDK137 & tar ${ }^{E E E E}$-eyfp & 20 & gift of David Kentner \\
\hline pDK138 & $\operatorname{tar}^{\mathrm{QQQQ}}$-eyfp & 20 & gift of David Kentner \\
\hline pVS138 & cheB-eyfp & 100 & [58] \\
\hline pDK19 & cheR-eyfp & 100 & {$[37]$} \\
\hline pDK159 & che $B^{S 164 C}$-eyfp & 50 & {$[40]$} \\
\hline pDK183 & $c h e B^{D 56 E}$-eyfp & 50 & gift of David Kentner \\
\hline pDK116 & eyfp-cheR $R^{D 154 A}$ & 50 & {$[40]$} \\
\hline
\end{tabular}

1:100 in $10 \mathrm{ml} \mathrm{TB}(10 \mathrm{~g} / \mathrm{l}$ tryptone, $5 \mathrm{~g} / \mathrm{l} \mathrm{NaCl}, \mathrm{pH} 7.0)$ containing appropriate antibiotics and inducers (Table 1 ). After growing at $34^{\circ} \mathrm{C}$ with $275 \mathrm{rpm}$ to $\mathrm{OD}_{600} \approx 0.45-$ 0.5 cells were two times washed in tethering buffer (10 $\mathrm{mM} \mathrm{KH} \mathrm{PO}_{4} / \mathrm{K}_{2} \mathrm{HPO}_{4}, 0.1 \mathrm{mM}$ EDTA, $10 \mathrm{mM}$ sodium lactate, $67 \mathrm{mM} \mathrm{NaCl}, 1 \mu \mathrm{M}$ methionine, $\mathrm{pH} 7.0$ ). To minimize growth and protein production, cells were subsequently incubated for at least $1 \mathrm{~h}$ at $4^{\circ} \mathrm{C}$.

\section{FRAP Analyses and data processing}

For FRAP experiments cells were immobilized on (poly) L-lysine-coated coverslips for $5 \mathrm{~min}$. Measurements were usually performed at $20^{\circ} \mathrm{C}(\mathrm{RT})$ or when indicated at $39^{\circ} \mathrm{C}$. For that, slides were placed in a metal chamber connected to a water bath. Cells were visualized with the $63 \times$ oil objective of a laser-scanning confocal microscope (Leica TCS SP2). Fluorescent cells were scanned by the $514 \mathrm{~nm}$ laser line of a $20 \mathrm{~mW}$ argon laser with $1-5 \%$ intensity and detected within $525-650 \mathrm{~nm}$ at $32-$ fold magnification. Regions of interest (ROIs) were bleached with two $0.336 \mathrm{~s}$ laser scans at $50 \%$ laser intensity using the same laser line. The following image series were recorded (Leica Confocal software, Version 2.61) by bidirectional scanning: one prebleach- and 10 postbleach images every $0.336 \mathrm{~s}, 10$ postbleach images every $3 \mathrm{~s}$ and depending on protein 10-40 postbleach images every $30 \mathrm{~s}$.

Images were analyzed by using a custom-written plugin [37] for ImageJ software, Version 1.34l (W. Rasband, National Institutes of Health, Bethesda, MD; http://rsb. info.nih.gov/ij). For FRAP evaluation, the polar region was defined as 52 pixles, which is approximately $20 \%$ of the average cell length. Fluorescence of the ROI was normalized two times: first to the fluorescence of the entire cell in the same image to compensate for gradual bleaching during scanning, second to the prebleach value of the ROI, to make different experiments comparable. To reduce variability that arises due to varying depth of bleaching, for experiments shown in Figure 1 and $3 \mathrm{~d}$ the value of the first post-bleach point was 
additionally subtracted and the curves were renormalized. Data were processed using KalaidaGraph software, Version 3.6 (Synergy Software).

For data fitting in Figure 2, protein exchange at chemotaxis clusters can be treated as a combination of anomalous diffusion and an exponential decay with the characteristic exchange time $\tau_{o b s}$ and fit with the following equation:

$$
I(t)=\frac{F_{0}+F_{\infty}\left(\frac{t}{t_{1 / 2}}\right)^{\alpha}}{1+\left(\frac{t}{t_{1 / 2}}\right)^{\alpha}}+C\left(1-e^{-\left[\frac{t}{\tau_{o b s}}\right]}\right),
$$

where $F_{0}$ accounts for the relative fluorescence intensity of free fluorescent protein after bleaching, $F_{\infty}$ is the corresponding intensity after recovery, $t_{1 / 2}$ is half-time of recovery, $\alpha$ is the factor accounting for anomalous diffusion and $C$ is the relative steady-state concentration of cluster-bound fluorescent protein [37]. Values for $t_{1 / 2}$ and $\alpha$ has been taken from a previous study, where diffusion of the same proteins were measured in a strain without chemotaxis clusters [37].

\section{Soft agar chemotaxis assays}

To test chemotaxis-driven spreading of MG1655, W3110 and RP437 on soft agar plates, $3 \mu \mathrm{l}$ of an overnight culture grown in TB were dropped on soft agar plates (TB, $0.3 \%$ agar) and incubated for 5 hours at either $34^{\circ} \mathrm{C}, 37^{\circ} \mathrm{C}, 39^{\circ} \mathrm{C}$ or $42^{\circ} \mathrm{C}$. Pictures were taken, swarm ring diameters were analyzed by ImageJ software and plotted using KalaidaGraph software.

\section{Immunoblotting}

Immunoblotting was performed as previously described [44]. Cells were grown as described above to give the same $\mathrm{OD}_{600}$ for all strains, washed and collected by centrifugation, resuspedend in Laemmli buffer and lysed for $10 \mathrm{~min}$ at $95^{\circ} \mathrm{C}$. Samples were separated on the $8 \%$ SDSpolyacrylamide gel and analyzed using primary polyclonal $\alpha$ Tar antibody at 1:5,000 dilution and IRDye 800 conjugated secondary antibody (Rockland) at 1:10,000 dilution. Note that $\alpha$ Tar antibody, which was raised against conserved signaling domain of receptor, recognizes other chemoreceptors with similar specificity. Membranes were scanned with an Odyssey Imager (LI-COR).

\section{Additional material}

Additional file 1: Figure S1. Modification levels of chemoreceptors in strains used for FRAP. The figure shows levels of chemoreceptor modification in strains expressing CheR and CheB fusions, determined by immunoblotting with receptor-specific antibodies.

\section{Acknowledgements}

We thank David Kentner for the kind gift of pDK137, pDK138 and pDK83 and Abiola Pollard for commenting on the manuscript. This work was supported by the Deutsche Forschungsgemeinschaft grant SO 421/3-3 and by the National Institutes of Health grant GM082938.

\section{Author details}

${ }^{1}$ Zentrum für Molekulare Biologie der Universität Heidelberg, DKFZ-ZMBH Alliance, Im Neuenheimer Feld 282, 69120 Heidelberg, Germany. ${ }^{2}$ BioQuant, Im Neuenheimer Feld 267, 69120 Heidelberg, Germany.

\section{Authors' contributions}

SS and KG performed all experiments and participated in the design of the study and in writing the manuscript. VS conceived the study, participated in its design and wrote the manuscript. All authors read and approved the final manuscript.

Received: 6 June 2011 Accepted: 6 October 2011 Published: 6 October 2011

\section{References}

1. Hazelbauer GL, Lai WC: Bacterial chemoreceptors: providing enhanced features to two-component signaling. Curr Opin Microbiol 2010, 13:124-132.

2. Sourjik V, Armitage JP: Spatial organization in bacterial chemotaxis. EMBO J 2010, 29:2724-2733.

3. Borkovich KA, Alex LA, Simon Ml: Attenuation of sensory receptor signaling by covalent modification. Proc Natl Acad Sci USA 1992, 89:6756-6760.

4. Bornhorst JA, Falke JJ: Attractant regulation of the aspartate receptorkinase complex: Limited cooperative interactions between receptors and effects of the receptor modification state. Biochemistry 2000, 39:9486-9493.

5. Endres RG, Oleksiuk O, Hansen CH, Meir Y, Sourjik V, Wingreen NS: Variable sizes of Escherichia coli chemoreceptor signaling teams. Mol Syst Biol 2008, 4:211.

6. Levit MN, Stock JB: Receptor methylation controls the magnitude of stimulus-response coupling in bacterial chemotaxis. J Biol Chem 2002, 277:36760-36765.

7. Li G, Weis RM: Covalent modification regulates ligand binding to receptor complexes in the chemosensory system of Escherichia coli. Cell 2000, 100:357-365

8. Sourjik V, Berg HC: Receptor sensitivity in bacterial chemotaxis. Proc Nat/ Acad Sci USA 2002, 99:123-127.

9. Amin DN, Hazelbauer GL: Chemoreceptors in signalling complexes: shifted conformation and asymmetric coupling. Mol Microbiol 2010, 78:1313-1323.

10. Alon U, Surette MG, Barkai N, Leibler S: Robustness in bacterial chemotaxis. Nature 1999, 397:168-171.

11. Amin DN, Hazelbauer GL: The chemoreceptor dimer is the unit of conformational coupling and transmembrane signaling. J Bacterio/ 2010, 192:1193-1200

12. Mello BA, Tu Y: Perfect and near-perfect adaptation in a model of bacterial chemotaxis. Biophys J 2003, 84.

13. Anand GS, Goudreau PN, Stock AM: Activation of methylesterase CheB: evidence of a dual role for the regulatory domain. Biochemistry 1998, 37:14038-14047.

14. Lan G, Schulmeister S, Sourjik V, Tu Y: Adapt locally and act globally: strategy to maintain high chemoreceptor sensitivity in complex environments. Mol Syst Biol 2011, 7:475.

15. Clausznitzer D, Oleksiuk O, Lovdok L, Sourjik V, Endres RG: Chemotactic response and adaptation dynamics in Escherichia coli. PLoS Comput Biol 2010, 6:e1000784.

16. Boldog T, Grimme S, Li M, Sligar SG, Hazelbauer GL: Nanodiscs separate chemoreceptor oligomeric states and reveal their signaling properties. Proc Natl Acad Sci USA 2006, 103:11509-11514.

17. Li M, Khursigara CM, Subramaniam S, Hazelbauer GL: Chemotaxis kinase CheA is activated by three neighbouring chemoreceptor dimers as effectively as by receptor clusters. Molecular microbiology 2011, 79:677-685. 
18. Li M, Hazelbauer GL: Core unit of chemotaxis signaling complexes. Proc Natl Acad Sci USA 2011, 108:9390-9395.

19. Maddock JR, Shapiro L: Polar location of the chemoreceptor complex in the Escherichia coli cell. Science 1993, 259:1717-1723.

20. Sourjik V, Berg HC: Localization of components of the chemotaxis machinery of Escherichia coli using fluorescent protein fusions. $\mathrm{Mol}$ Microbiol 2000, 37:740-751.

21. Greenfield D, McEvoy AL, Shroff H, Crooks GE, Wingreen NS, Betzig E, Liphardt J: Self-organization of the Escherichia coli chemotaxis network imaged with super-resolution light microscopy. PLOS Biol 2009, 7: e1000137.

22. Briegel A, Ding HJ, Li Z, Werner J, Gitai Z, Dias DP, Jensen RB, Jensen GJ: Location and architecture of the Caulobacter crescentus chemoreceptor array. Mol Microbiol 2008, 69:30-41.

23. Briegel A, Ortega DR, Tocheva El, Wuichet K, Li Z, Chen S, Muller A, lancu CV, Murphy GE, Dobro MJ, et al: Universal architecture of bacterial chemoreceptor arrays. Proc Natl Acad Sci USA 2009, 106:17181-17186

24. Khursigara CM, Wu X, Subramaniam S: Chemoreceptors in Caulobacter crescentus: trimers of receptor dimers in a partially ordered hexagonally packed array. J Bacteriol 2008, 190:6805-6810

25. Kim KK, Yokota H, Kim SH: Four-helical-bundle structure of the cytoplasmic domain of a serine chemotaxis receptor. Nature 1999, 400:787-792.

26. Ames P, Studdert CA, Reiser RH, Parkinson JS: Collaborative signaling by mixed chemoreceptor teams in Escherichia coli. Proc Natl Acad Sci USA 2002, 99:7060-7065.

27. Kentner D, Thiem S, Hildenbeutel M, Sourjik V: Determinants of chemoreceptor cluster formation in Escherichia coli. Mol Microbiol 2006, 61:407-417.

28. Skidmore JM, Ellefson DD, McNamara BP, Couto MM, Wolfe AJ, Maddock JR: Polar clustering of the chemoreceptor complex in Escherichia coli occurs in the absence of complete CheA function. J Bacteriol 2000, 182:967-973.

29. Studdert CA, Parkinson JS: Insights into the organization and dynamics of bacterial chemoreceptor clusters through in vivo crosslinking studies. Proc Natl Acad Sci USA 2005, 102:15623-15628.

30. Gestwicki JE, Kiessling LL: Inter-receptor communication through arrays of bacterial chemoreceptors. Nature 2002, 415:81-84.

31. Lai RZ, Manson JM, Bormans AF, Draheim RR, Nguyen NT, Manson MD: Cooperative signaling among bacterial chemoreceptors. Biochemistry 2005, 44:14298-14307.

32. Sourjik V, Berg HC: Functional interactions between receptors in bacterial chemotaxis. Nature 2004, 428:437-441.

33. Vaknin A, Berg HC: Physical responses of bacterial chemoreceptors. J Mol Biol 2007, 366:1416-1423.

34. Cantwell BJ, Draheim RR, Weart RB, Nguyen C, Stewart RC, Manson MD: CheZ phosphatase localizes to chemoreceptor patches via CheA-short. J Bacteriol 2003, 185:2354-2361

35. Banno S, Shiomi D, Homma M, Kawagishi I: Targeting of the chemotaxis methylesterase/deamidase CheB to the polar receptor-kinase cluster in an Escherichia coli cell. Mol Microbiol 2004, 53:1051-1063.

36. Shiomi D, Zhulin IB, Homma M, Kawagishi I: Dual recognition of the bacterial chemoreceptor by chemotaxis-specific domains of the CheR methyltransferase. J Biol Chem 2002, 277:42325-42333.

37. Schulmeister $S$, Ruttorf $M$, Thiem S, Kentner D, Lebiedz D, Sourjik V. Protein exchange dynamics at chemoreceptor clusters in Escherichia coli. Proc Natl Acad Sci USA 2008, 105:6403-6408.

38. Barnakov AN, Barnakova LA, Hazelbauer GL: Efficient adaptational demethylation of chemoreceptors requires the same enzyme-docking site as efficient methylation. Proc Natl Acad Sci USA 1999, 96:10667-10672.

39. Wu J, Li J, Li G, Long DG, Weis RM: The receptor binding site for the methyltransferase of bacterial chemotaxis is distinct from the sites of methylation. Biochemistry 1996, 35:4984-4993.

40. Kentner D, Sourjik V: Dynamic map of protein interactions in the Escherichia coli chemotaxis pathway. Mol Syst Biol 2009, 5:238.

41. Li J, Swanson RV, Simon MI, Weis RM: The response regulators CheB and CheY exhibit competitive binding to the kinase CheA. Biochemistry 1995 34:14626-14636.

42. Shrout AL, Montefusco DJ, Weis RM: Template-directed assembly of receptor signaling complexes. Biochemistry 2003, 42:13379-13385.
43. Erbse AH, Falke JJ: The core signaling proteins of bacterial chemotaxis assemble to form an ultrastable complex. Biochemistry 2009, 48:6975-6987.

44. Oleksiuk O, Jakovljevic V, Vladimirov N, Carvalho R, Paster E, Ryu WS, Meir Y, Wingreen NS, Kollmann M, Sourjik V: Thermal robustness of signaling in bacterial chemotaxis. Cell 2011, 145:312-321.

45. Kollmann M, Løvdok L, Bartholome K, Timmer J, Sourjik V: Design principles of a bacterial signalling network. Nature 2005, 438:504-507.

46. Barnakov AN, Barnakova LA, Hazelbauer GL: Allosteric enhancement of adaptational demethylation by a carboxyl-terminal sequence on chemoreceptors. J Biol Chem 2002, 277:42151-42156.

47. Adler J, Templeton B: The effect of environmental conditions on the motility of Escherichia coli. J Gen Microbiol 1967, 46:175-184.

48. Bethani I, Skanland SS, Dikic I, Acker-Palmer A: Spatial organization of transmembrane receptor signalling. EMBO J 2010, 29:2677-2688.

49. Kim SH, Wang W, Kim KK: Dynamic and clustering model of bacterial chemotaxis receptors: structural basis for signaling and high sensitivity. Proc Natl Acad Sci USA 2002, 99:11611-11615.

50. Liberman L, Berg HC, Sourjik V: Effect of chemoreceptor modification on assembly and activity of the receptor-kinase complex in Escherichia coli. J Bacteriol 2004, 186:6643-6646.

51. Shiomi D, Banno S, Homma M, Kawagishi I: Stabilization of polar localization of a chemoreceptor via its covalent modifications and its communication with a different chemoreceptor. J Bacteriol 2005, $187: 7647-7654$

52. Meir Y, Jakovljevic V, Oleksiuk O, Sourjik V, Wingreen NS: Precision and kinetics of adaptation in bacterial chemotaxis. Biophys J 2010, 99:2766-2774.

53. Korobkova E, Emonet T, Vilar JM, Shimizu TS, Cluzel P: From molecular noise to behavioural variability in a single bacterium. Nature 2004 428:574-578.

54. Emonet T, Cluzel P: Relationship between cellular response and behavioral variability in bacterial chemotaxis. Proc Natl Acad Sci USA 2008, 105:3304-3309.

55. Matthaus F, Jagodic M, Dobnikar J: E. coli superdiffusion and chemotaxissearch strategy, precision, and motility. Biophys J 2009, 97:946-957.

56. Parkinson JS, Houts SE: Isolation and behavior of Escherichia coli deletion mutants lacking chemotaxis functions. J Bacteriol 1982, 151:106-113.

57. Amann $E$, Ochs B, Abel KJ: Tightly regulated tac promoter vectors useful for the expression of unfused and fused proteins in Escherichia coli. Gene 1988, 69:301-315.

58. Lovdok L, Kollmann M, Sourjik V: Co-expression of signaling proteins improves robustness of the bacterial chemotaxis pathway. J Biotechnol 2007, 129:173-180

doi:10.1186/1471-2180-11-222

Cite this article as: Schulmeister et al:: Effects of receptor modification and temperature on dynamics of sensory complexes in Escherichia coli chemotaxis. BMC Microbiology 2011 11:222.

\section{Submit your next manuscript to BioMed Central and take full advantage of:}

- Convenient online submission

- Thorough peer review

- No space constraints or color figure charges

- Immediate publication on acceptance

- Inclusion in PubMed, CAS, Scopus and Google Scholar

- Research which is freely available for redistribution

Submit your manuscript at www.biomedcentral.com/submit
C Biomed Central 